\title{
Wie gut helfen Spray und Spülung bei chronischer Sinusitis?
}

\begin{abstract}
Patienten mit chronischer Sinusitis wird empfohlen, die Nase mit Salzlösung zu spülen oder Nasensprays mit Antibiotika-, Kortikosteroidoder Fungizidzusatz zu verwenden, in der Hoffnung, damit die mukoziliare Clearance zu verbessern sowie Entzündungsprozesse und Infektionen zu stoppen. Zur Wirksamkeit gehen die Meinungen allerdings auseinander.
\end{abstract}

Inwieweit topische Anwendungen bei chronischer Sinusitis tatsächlich empfehlenswert sind, untersuchten US-amerikanische Mediziner anhand der verfügbaren Literatur: Für das Spülen mit Salzlösung zeichnete sich die beste Evidenz ab. In insgesamt 16 randomisierten kontrollierten Studien fanden Calvin C. Wei vom St. Luke Hospital in New York und seine Kol- legen den Nutzen der nasalen Anwendung von Salzlösung bestätigt. Als eigenständiges Behandlungsverfahren sowie ergänzend nach endoskopischer Sinuschirurgie milderte es effektiv die Beschwerden.

\section{Kortikosteroidtherapie ließ \\ Polypen schrumpfen}

Die lokale Kortikosteroidtherapie war noch intensiver erforscht worden, insgesamt 25 Studien hatten randomisiert und kontrolliert den Nutzen überprüft. Eindeutig von Vorteil war eine lokale Kortikosteroidbehandlung aber nur, wenn infolge der chronischen Nebenhöhlenentzündung Polypen entstanden waren, die unter Therapie wieder schrumpften.

Eher dürftig fiel die Beweislage zur Antibiotika- und Fungizidtherapie aus: Ganze zwei randomisierte kontrollierte Studien existierten zur topischen Antibi- ose und vier zur topischen Pilzbehandlung, wobei meist der Wirkstoff Amphotericin $B$ getestet wurde. Ein eindeutiger Vorteil bestätigte sich weder für das Antibiotika- noch für das Fungizidspray. Die mit dem Pilzmittel behandelten Patienten schnitten nicht besser ab als Patienten, die ihre Nasenhöhle mit einem Salzwasserspray befeuchtet hatten.

Als sinnvoll erachten die Studienautoren das Spülen mit Salzlösung. Der Nutzen topischer Antibiotika- und Kortikosteroidanwendungen hingegen müsse noch genauer untersucht werden. Die Studienautoren sind sich aber sicher, dass beides künftig an Bedeutung gewinnen wird, nicht zuletzt aufgrund der Nebenwirkungen einer systemischen Antibiotika- oder Kortikosteroidtherapie.

$(d k)$

Wei CC et al, Laryngoscope 2013 (online first)

\section{Risiko Reißverschluss}

\section{Hinterm Hosenlatz lauert der Schmerz}

\begin{abstract}
Darüber, dass die Hosen keine Knöpfe mehr haben, ist schon manche Träne vergossen worden vor allem von jenen, die sich ihre Vorhaut im Reißverschluss eingeklemmt haben. Malheurs dieser Art sind indes weder lustig noch besonders selten.
\end{abstract}

Obwohl der Schieber seit mehr als 100 Jahren über die Krampen gleitet, gab es zur Inzidenz Reißverschluss-verursachter Genitalläsionen bis vor kurzem keine verlässlichen Zahlen. US-Urologen um Herman Bagga, San Francisco, haben diesem Mangel nun abgeholfen. Anhand der Daten, die von der US-Verbraucherschutzkommission CPSC vorgehalten werden, schätzten Bagga und Kollegen die Zahl der Penisverletzungen durch Reißverschlüsse in den Jahren 2002 bis
2010 auf USA-weit rund 18.000. Knapp $30 \%$ aller Penisläsionen von Erwachsenen lag der Fehlgebrauch eines Zippverschlusses zugrunde, was Platz eins in der Ursachenstatistik bedeutete. Im pädiatrischen Kollektiv erreichte die Quote circa $17 \%$. Nur von Quetschungen des Penis durch Toilettenbrillen wurden Knaben noch häufiger ereilt.

\section{Riskante Ratschläge}

Weniger als 1\% der Verletzungen durch Reißverschlüsse betrafen das Skrotum. Rein anekdotischen Charakter hatten Unfälle mit Zippern im Genitalbereich, in die Frauen verwickelt worden waren.

Ebenfalls nicht risikofrei sind die Ratschläge, wie eingezwängtes Gewebe zu befreien sei. Ob das nötige Feingefühl in jedem Fall walten kann, lässt sich mit Blick auf das in der Fachliteratur empfoh-

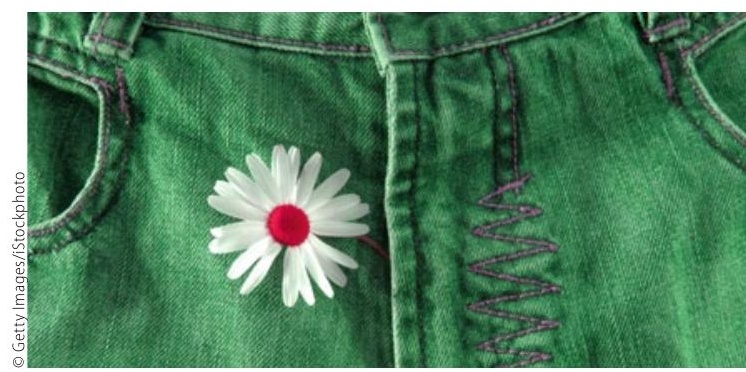

lene Instrumentarium - von Schraubenziehern und Zangen über Saitenschneider bis zu Drahtscheren - mit Fug bezweifeln. In therapierefraktären Fällen gilt die Zirkumzision als Ultima Ratio.

„Die meisten der Verletzungen ereignen sich unabsichtlich beim Schließen des Zippers", notieren Bagga und sein Team.

Bagga HS et al, BJU Int 2013 (online first) 